\title{
Methodology for Assessing Adaptive Cruise Control Behavior
}

\author{
Zevi Bareket, Paul S. Fancher, Huei Peng, Kangwon Lee, and Charbel A. Assaf
}

\begin{abstract}
This paper reports on nonintrusive methods for characterizing the longitudinal performance of vehicles equipped with adaptive cruise control (ACC) systems. It reports the experimental set-up and procedures for measuring ACC system performance, followed by the modeling and simulation of the measured ACC performance. To further assess the interaction of ACC vehicles with human-controlled traffic, microscopic simulation involving both a human-driver model and an ACC model is discussed.
\end{abstract}

Index Terms-Adaptive cruise control (ACC), clearance, global positioning system (GPS), range measurement, system identification, time gap, traffic flow.

\section{INTRODUCTION}

A CC systems are currently being introduced into the consumer market by vehicle manufacturers. These systems have been tuned to obtain high levels of acceptance from individual consumers. Results from initial operational tests [1] indicate that drivers generally like the comfort and convenience provided by systems that control the time gap between vehicles. However, there is concern that the aggregate dynamic effects from many such vehicles in traffic could act to disrupt flow.

This paper presents research work whose ultimate goal is to address the influence of the characteristics of adaptive cruise control (ACC) systems on the behavior of vehicles in traffic streams [2]. The scope of the research includes the testing of different vehicles that are currently being sold to the public by car manufacturers. These vehicles have been instrumented and operated in order to identify system properties that are important to strings of closely spaced vehicles. The measurements have been done in a manner that is independent of the actual ACC hardware installed in the vehicles by the manufacturers.

As a step toward the ultimate goal of predicting the influence of ACC on traffic flow, results from a pre-determined set of headway maneuvers have been used to identify parameters and to characterize vehicle performance. The models deduced from the test results are intended to provide the basis for computerbased studies of traffic flow. These studies will be expected to provide preliminary results indicating the predicted performance of ACC vehicles similar to those available on the market

Manuscript received August 29, 2002; revised October 2, 2003.

Z. Bareket and P. S. Fancher are with the University of Michigan Transportation Research Institute (UMTRI), Ann Arbor, MI 48109-2150 USA (e-mail: bareket@umich.edu).

H. Peng and K. Lee are with the Mechanical Engineering and Applied Mechanics Department, University of Michigan, Ann Arbor, MI 48109-2121 USA

C. A. Assaf was with the Mechanical Engineering and Applied Mechanics Department, University of Michigan, Ann Arbor, MI 48109-2121 USA. He is now with the Robert Bosch Corporation, Farmington Hills, MI 48331-3417 USA.

Digital Object Identifier 10.1109/TITS.2003.821288 now. In this context, special traffic simulation models that incorporate various numbers of manual and ACC-controlled vehicles have been used to predict the influence of ACC systems on traffic flow [3].

\section{Problem Statement}

Vehicle manufacturers have developed ACC systems that control the driving and braking systems of their vehicles. The primary functional purpose of these systems is to maintain a prescribed time-gap relationship between the ACC-equipped vehicle and its immediately preceding vehicle, when one is present.

In addition to the controls associated with conventional cruise control, the driver is also provided with a control that may be used to select a desired time-gap relationship which has maximum and minimum values as pre-determined by the designer of the ACC system. Using these controls, a driver may select a free-driving speed (the "set speed") and a time-gap relationship to fit the individual's driving preferences. Subjective ratings from field tests indicate that many drivers enjoy using these types of systems [1].

Fig. 1 provides a simplified technical portrayal of the basic one-on-one driving situation considered in the design of current ACC systems.

Where $V_{p}$ is the speed of the preceding vehicle, $R$ is the range clearance between the two vehicles, $V$ is the speed of the ACC vehicle, and $\dot{R}$ is the range rate, as indicated by

$$
\dot{R}=V_{p}-V \text {. }
$$

The driving situations studied in this paper are extensions of the one-on-one driving situation portrayed in Fig. 1. That is, the formation of strings of such preceding/following vehicle pairs in the traffic stream. As market penetration increases, the possibility that several ACC-equipped vehicles will spontaneously form a string of consecutive vehicles also increases. This scenario raises issues concerning characteristics of ACC systems that are desirable in the context of the flow of traffic streams. Technical challenges that are addressed in this paper are:

1) to quantitatively assess the influences that current $\mathrm{ACC}$ systems might have on string behavior;

2) to determine those system characteristics that are favorable to good string performance.

Fig. 2 shows a string of vehicles in which the velocity of each following vehicle depends upon the velocity and range clearance relative to its immediately preceding vehicle. 


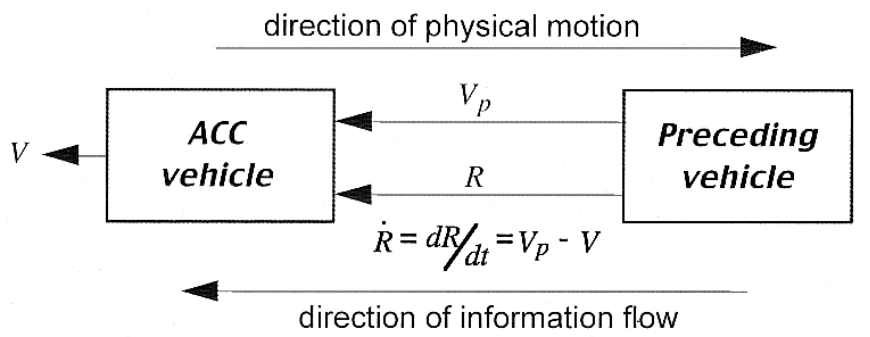

Fig. 1. One-on-one driving.

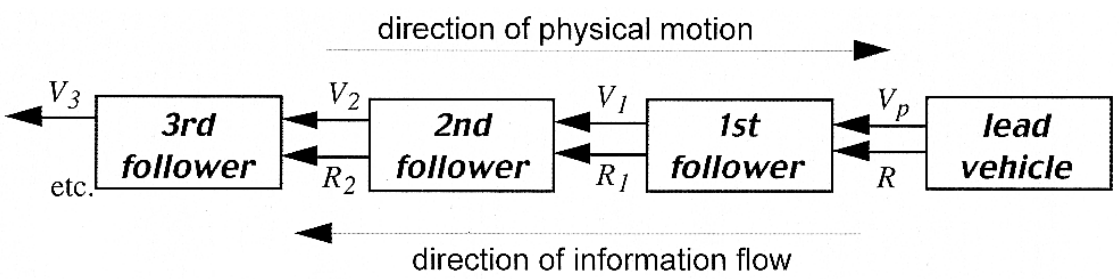

Fig. 2. Vehicle-string representation of a dense traffic stream.

Clearly, the string shown in Fig. 2 consists of a sequence of one-on-one situations. For the first follower, $V_{1}$ and $R_{1}$ depend upon $V_{p}$. In addition, for the next follower, $V_{2}$ and $R_{2}$ depend upon $V_{1}$, and so forth.

Examination of time histories from vehicle measurements may provide helpful insight into the meaning of observed responses. In the time domain, string performance can be characterized using experiments involving the effects of speed differences between the following vehicle and its immediately preceding vehicle. Heuristically, one can envision situations in which the lead-vehicle slows-down abruptly to a lower speed and during the ensuing transient each following vehicle slows-down more than its preceding vehicle. This means that each following vehicle undershoots the speed of its preceding vehicle before reaching the speed of the preceding vehicle at steady state. As a result, the minimum range clearance between successive vehicles decreases from vehicle to vehicle until a vehicle far enough back in the string has to stop completely in order to avoid colliding with its preceding vehicle. This leads to stop-and-go driving in proving grounds or staged experiments. Clearly, it represents poor string performance. For example see references [1], [4].

This paper describes methods that are being used to develop knowledge as needed for assuring that ACC systems will improve (or at least not degrade) string performance over what it is today with manual, unassisted driving. In this context, the situations and variables described in Figs. 1 and 2 apply to manual unassisted as well as ACC driving.

\section{ACC System Performance Measurement}

The basic element of the performance-prediction approach discussed here is an accurate model to predict how a given vehicle will respond to the motion of its preceding vehicle. Once this one-on-one phenomenon is represented in a model, a string model may be developed by placing from 2 to $\mathrm{N}$ vehicle system models in a sequence to make a simulation of a string of vehicles.
This approach involves the testing of individual ACC-equipped vehicles to capture their dynamic response to motion disturbances from a preceding vehicle. Response measurements of each ACC vehicle have been made in a nonintrusive fashion, i.e., the researchers had no access to data from any of the OEM-installed on-board systems - radar, throttle, engine, etc.

Essential data that had to be obtained during these tests were range clearance, range rate, and velocity of the ACC-equipped vehicle. Since access to the on-board data was not available, alternative methods for gathering the data had to be devised. The data acquisition package for each vehicle includes a differential GPS (DGPS). Clearly, these DGPS devices are for experimental purposes only, and they are not part of the ACC system.

GPS is typically used as a single-point data source to obtain the position, elevation, velocity, etc., of a single point of interest. By using two GPS units that operate independently, and by synchronizing their data, the relative position (i.e., range clearance) and relative velocity (i.e., range rate) between them can be determined. The velocity of each vehicle is obtained directly from the individual GPS units. This method provides range clearance determination using a system that is readily installed without invading the vehicle's ACC sensor and control systems.

Utilizing GPS as a measuring tool between two moving points raises two questions: 1) how accurately were range clearance and range-rate measured using this approach, and 2) how reliable and consistent were the data. To address these two questions, and thus verifying the validity of using GPS as a means to measure range clearance and range rate between two vehicles in this study, two tests were performed. First, two GPS antennas were mounted on the flat rooftop of a van trailer at a fixed distance of $13.55 \mathrm{~m}$ between them. The truck was then driven on the highway while collecting the GPS data.

Ideally, one would expect the range clearance data to show a horizontal line at the nominal value of $13.55 \mathrm{~m}$, and the range-rate data to show a horizontal line at $0 \mathrm{~m} / \mathrm{s}$. The real data are shown in Fig. 3. 

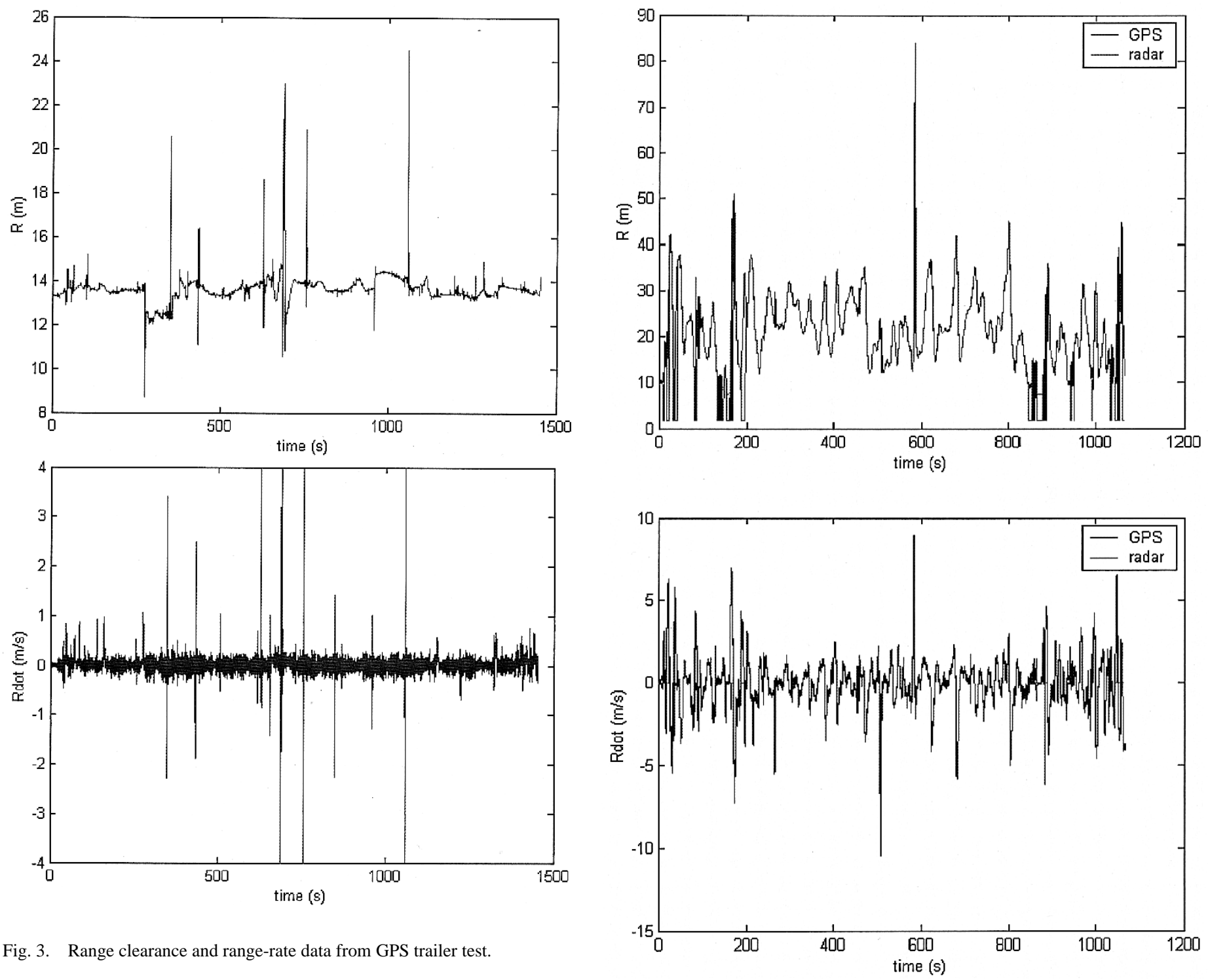

Fig. 3. Range clearance and range-rate data from GPS trailer test.

The figure shows that for the most part, the data obtained by using the GPS are in good proximity to the truth values of $13.55 \mathrm{~m}$ for range clearance, and $0 \mathrm{~m} / \mathrm{s}$ for range rate. However, the figure also shows that the application of GPS as a rangemeasuring means has some deficiencies. Three observations can readily be made.

1) The presence of very short, but high-magnitude data peaks.

2) If the high-frequency peaks are ignored, the data are bounded by approximately $\pm 1 \mathrm{~m}$ about the true nominal value.

3) Data variations can be abrupt (e.g., range clearance data at about 250 s. in Fig. 3 or smooth (e.g., the range clearance data at about $500 \mathrm{~s}$.).

Three factors mainly contribute to these deficiencies: bridges, change of satellites, and an asymmetrical reflection effect from the trailer's roof surface. As a result, the data contain unrealistic jumps.

The experiment described above showed that GPS can be used to validate range information between two antennas that are fixed relative to each other (though their common base was

Fig. 4. Range and range rate data from the two-car GPS test.

moving). The question that is being asked, however, is "How accurately can we assess range and range rate, if the two antennas are mounted on different vehicles that are moving relative to each other?" The following experiment was designed and performed to address that question.

In this experiment, one vehicle (designated as the lead car) carried the first GPS data system, and a second vehicle that was also equipped with a radar range sensor (designated as the following car), carried the second GPS data system. The two vehicles were then driven on the highway under normal traffic conditions. The data that were collected from both the radar sensor and the GPS were later analyzed and compared. Fig. 4 shows good agreement between the radar and the GPS data. The difference between the two range measurements was mostly bounded by $\pm 0.5 \mathrm{~m}$.

Once the validity of the GPS as a measuring tool was established, a test matrix was formed to explore those performance measures most likely to affect string performance. Initially, the test matrix for each vehicle was comprised of eight tests. 
1) Constant-speed following: Exercise all time-gap settings at highway speeds up to $70 \mathrm{mph}(110 \mathrm{kph})$ as well as low speeds down to $30 \mathrm{mph}(50 \mathrm{kph})$.

2) Changing time-gap: Exercise and characterize the transition when time-gap-setting changes are made while following.

3) Closing: The host car approaches the lead vehicle at $V_{\text {set }}>V_{p}$ and characterize the transition to constant-speed following.

4) U-Test: While following, the preceding vehicle decelerates, keeps the new speed, and then resumes the previous speed. During the actual testing, it was found that the extended length of time required for a successful test completion, was frequently unavailable: other cars cut in, the required test speed could not be maintained due to traffic flow, etc. The U-test was thus broken down to two parts: a "down-L" and an "up-L". These tests could be done on a proving grounds with long straight sections of approximately 3-4 km. Even so, the range-clearance response may be slow enough that steady following with $\dot{R}$ (range rate) approximately equal to zero will not be reached within $3 \mathrm{~km}$. Also, some ACC systems may have velocity-control rules that respond to road curvature (usually through yaw-rate consideration).

5) Wobbly: Low-amplitude, low-frequency speed oscillations by the preceding vehicle. This is essentially a test like the one described under "Constant-speed following" above, only that the lead vehicle was constantly changing its speed to enrich the frequency content of the data. Specifications on the amplitude could be enforced for this test, but an important idea in this application is to adjust for low frequency and for small amplitude characteristics that can be highly nonlinear (e.g., dead zones, etc.).

6) Cut-in: The preceding car cuts-in to position itself in front of the host vehicle.

7) Set speed above (cut-out): The preceding car leaves the path of the host vehicle, which resumes $V_{\text {set }}$.

8) Set speed below: Accelerator override to get above $V_{\text {set }}$ and release to let the system resume.

As the testing progressed, side-by-side with the data analysis, it became evident that some of the test maneuvers impact the system-identification process more than others. Also, certain maneuvers were not perceived as predominant in driving that is associated with strings of vehicles. Eventually, from the original list above, the research focused on acquiring large amounts of data for tests (1), (3), (4), (5), (7), and (8).

\section{IDENTIFICATION OF PERTINENT ACC-SYSTEM CHARACTERISTICS}

\section{A. The Model Used to Fit Data}

The GPS provided vehicle position and velocity information 10 times per second. This sampled data was stored on-board the vehicle and processed later using system-identification techniques to characterize each of the test vehicles. The model used for this purpose is a second-order state space model, with $V_{p}$ as input and $V$ and $R$ as outputs. The form of the model is:

$$
\left[\begin{array}{c}
V_{k+1} \\
R_{k+1}-R_{\min }
\end{array}\right]=\underbrace{\left[\begin{array}{ll}
a_{1} & a_{2} \\
a_{3} & a_{4}
\end{array}\right]}_{A} \cdot\left[\begin{array}{c}
V_{k} \\
R_{k}-R_{\min }
\end{array}\right]+\underbrace{\left[\begin{array}{l}
b_{1} \\
b_{2}
\end{array}\right]}_{B} \cdot V_{p_{k}}
$$

where $k$ is the discrete-time sampling index. The model includes a minimum range clearance $\left(R_{\min }\right)$ that some manufacturers use in their systems. From (1), and by using the forward differentiation approximation, we get:

$$
R_{k+1} \approx R_{k}+T_{s} \cdot\left(V_{p_{k}}-V_{k}\right)
$$

i.e., $a_{3} \approx-T_{s}, a_{4} \approx 1$, and $b_{2} \approx T_{s}$. We found that these theoretically-derived parametric values for $a_{3}, a_{4}$, and $b_{2}$ match those found by the system-identification algorithms. Hence, only three parameters $\left(a_{1}, a_{2}\right.$, and $\left.b_{1}\right)$ remained to be estimated. The simple model shown above was found to be accurate enough. Therefore, higher-order models were not used. The identification procedure uses built-in numerical algorithms such as PEM and N4SID that are built into Matlab ${ }^{1}$ 6.0 .

\section{B. Switching Rules for Driving Scenarios}

ACC designers typically use more than one control rule for their ACC-equipped vehicles. The reason is that driving situations vary, and thus require that the ACC will respond differently. For example, in a down-L maneuver, the following car cannot predict what the preceding car is doing. The car has to react quickly and then its velocity may overshoot. The same car, in a closing situation, will detect the slower preceding car further ahead, it can "plan" its closing and thus the velocity has little or no overshoot. Again, the same car in a cut-in situation may need to brake hard (emergency braking) to avoid a collision.

These different behaviors are difficult to model using a single linear system. Therefore, switching rules have been included in the car models. These rules are not necessarily the same rules used by the manufacturers. The number of possibilities is large, and re-formulating the exact one is practically impossible. Nevertheless, switching rules that approximate the vehicles' behavior in the different tests have been developed.

After examining the data, the range-range rate diagram was divided into six control regions: free flowing (conventional cruise control), up L, down L, closing, wobbly and emergency braking (e.g., cut in). The model does not know a-priori what type of test it will deal with. That is, it does not know that the preceding car will do a down L. For example, it may start with the wobbly model (the default model for a following case) and then estimate online that this is a down L situation and switches to the corresponding region model. Fig. 5 illustrates the different control regions and the switching boundaries.

The switching from one control region to another is not always defined in terms of boundaries. For example, "Closing" and "Down L" occupy the same range-range rate space in

\footnotetext{
${ }^{1}$ Matlab is a registered trademark of The MathWorks, Natick, MA.
} 


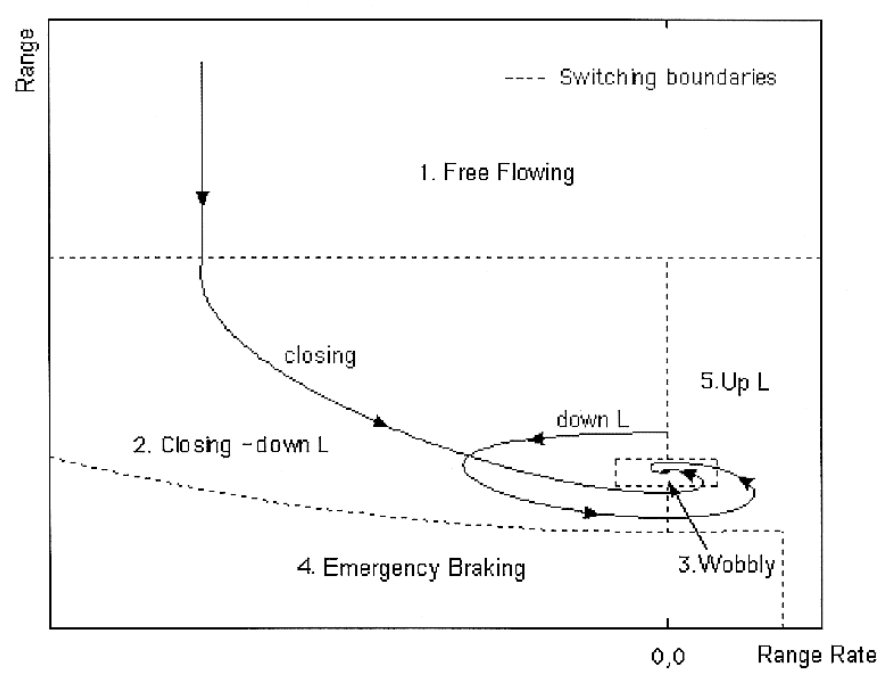

Fig. 5. Mapping the six regions into the range-versus-range-rate space.

TABLE I

THE RMS VALUES OF THE FIT

\begin{tabular}{|c|c|c|c|c|c|c|c|}
\hline & & Free Flou & Closing & Down L & Up L & Wobbly & $\begin{array}{l}\text { Emergency } \\
\text { Braking }\end{array}$ \\
\hline \multirow[t]{2}{*}{ Car A } & $V$ & 0.19 & 0.23 & 0.19 & 0.14 & 0.21 & 0.34 \\
\hline & $R$ & $\mathrm{~N} / \mathrm{A}$ & 1.27 & 1.26 & 0.97 & 1.02 & 1.76 \\
\hline \multirow[t]{2}{*}{ Car B } & $V$ & 0.44 & 0.27 & 0.42 & 0.30 & 0.12 & 0.32 \\
\hline & $R$ & N/A & 1.64 & 2.08 & 2.16 & 1.94 & 1.91 \\
\hline \multirow[t]{2}{*}{$\operatorname{Car} \mathrm{C}$} & $V$ & 0.39 & 0.19 & 0.15 & 0.13 & 0.21 & 0.47 \\
\hline & $R$ & $\mathrm{~N} / \mathrm{A}$ & 1.08 & 0.77 & 0.92 & 1.16 & 4.43 \\
\hline
\end{tabular}

Units for $V$ are $\mathrm{m} / \mathrm{sec}$., units for $R$ are $\mathrm{m}$.

Fig. 5. To determine which is the "effective" control region, the model uses the preceding situation: if the model entered the "Closing/Down-L" space from the "Free Flowing" control region, it will then be considered in the "Closing" region; On the other hand, if it entered that space from the "Wobbly" or the "Emergency Braking" control regions, it be considered in the "Down L" control region. Also, to go from "Wobbly" to "Down L" or "Up L", the system needs to detect a change in $V_{p}$ higher than a certain rate for a sustained period of time. Note, also that the "Wobbly" box is centered at the equilibrium point: (desired range rate $=0$, desired range clearance $=T_{h} \cdot V_{p}+R_{\min }$, where $T_{h}$ is the desired time-gap in seconds). Thus the location of "Wobbly" region changes with $V_{p}$.

\section{Quality of Fit}

In general, the matrices A and B [see (2)] were determined numerically by a least-square analysis. Most of the test scenarios are repeatable and thus certain "averaging" makes sense. However, one of the cars tested was very nonlinear in its behavior. Therefore, instead of "averaging," the most representative repetition was chosen. Table I shows the errors between the simulated and the measured data. These errors are averaged on all the repetitions in each case, except for the "Wobbly" case.
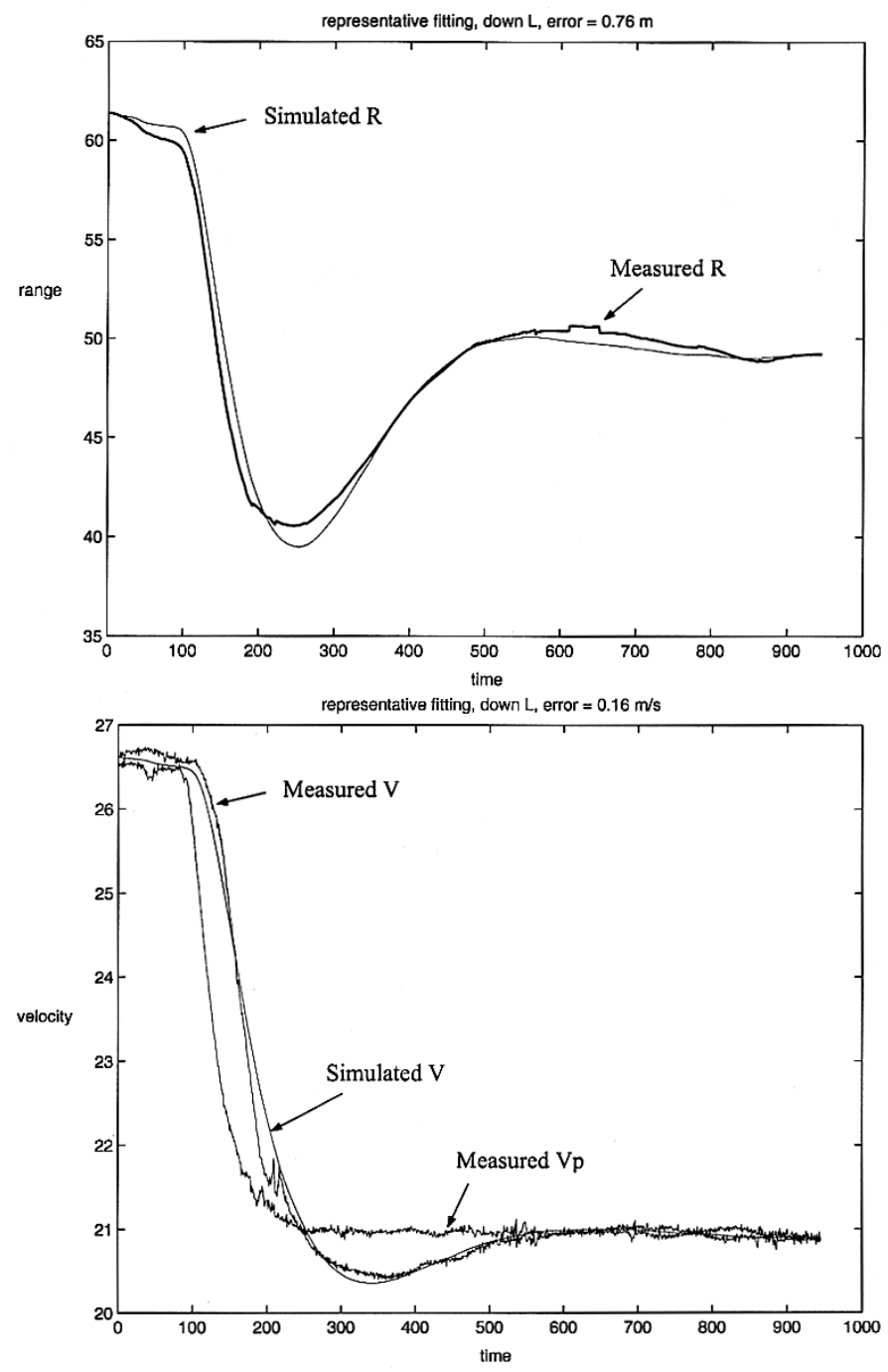

Fig. 6. Representative data-fitting results for a down-L situation.

By comparing the rms errors of the data fitting to the GPS accuracy, we found the quality of the fit to be satisfactory. Notice that car B has slightly worse results than the two others because of its very nonlinear algorithm.

Fig. 6 shows representative model-fitting results for a down-L test. Apparently, the model prediction and test results are qualitatively very similar.

\section{Procedures for Evaluating String Performance}

\section{A. Representative Cars}

Parametric values that are suitable for representing each specific car were derived. These results are proprietary information that the vehicle manufacturers do not want to share. However, since the purpose of this study is not to evaluate each manufacturer's ACC algorithm, three representative ACC systems were created for use in studying parametric variations that are representative of the differences in production vehicles. These representative systems differ in their responsiveness: "quick" indicates the use of higher acceleration rates and "slow" indicates the use of smaller acceleration rates when compared to those 

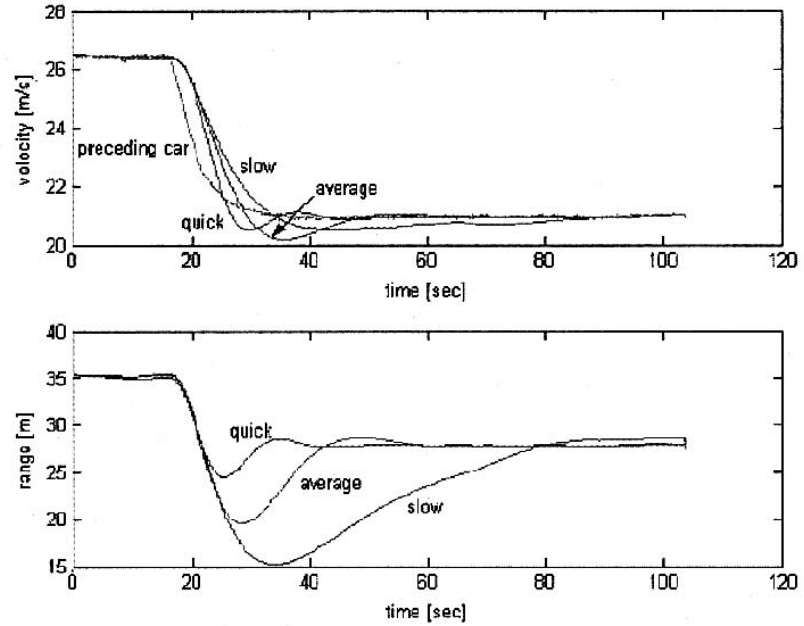

(a)
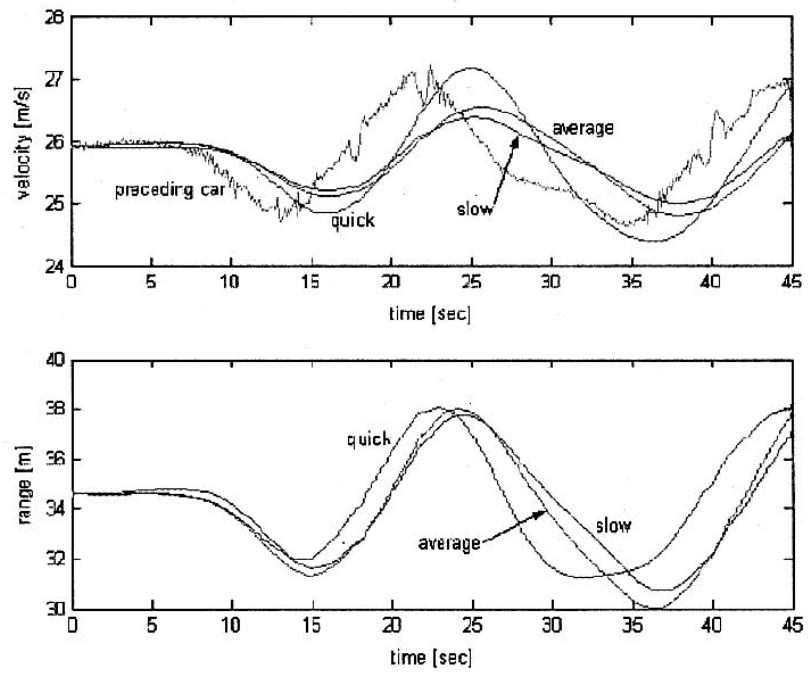

(c)

Fig. 7. Predicitve response (a) closing, (b) down L, (c) wobbly and (d) cut-in

rates approximating the median levels of acceleration observed in the manufacturer's vehicles.

Fig. 7 shows the simulated responses of these representative cars. One can see that the average ACC car overshoots in range clearance more than the quick $\mathrm{ACC}$ vehicle, and the slow ACC overshoots even more. For the average ACC car $T_{h}$ is $1.33 \mathrm{~s}$, which is close to the human driver's desired time-gap at highway speeds in light to medium traffic.

These representative cars can be simulated to operate together as a string. The string can be comprised of any combination of representative vehicles. Fig. 8 shows, for example, results of a string consisting of a leader plus three identical following vehicles ("Average" type) performing a down-L maneuver. Clearly, the overshoot in vehicle speed and range clearance amplifies along the string. Although there appears to be only a moderate problem for this four-car string, it is clear that, if the string size is increased, the range clearance between vehicles would eventually approach zero. Clearly, the string performance of the "Average" ACC vehicle could lead to stop-and-go situations. This is an issue to be studied carefully.
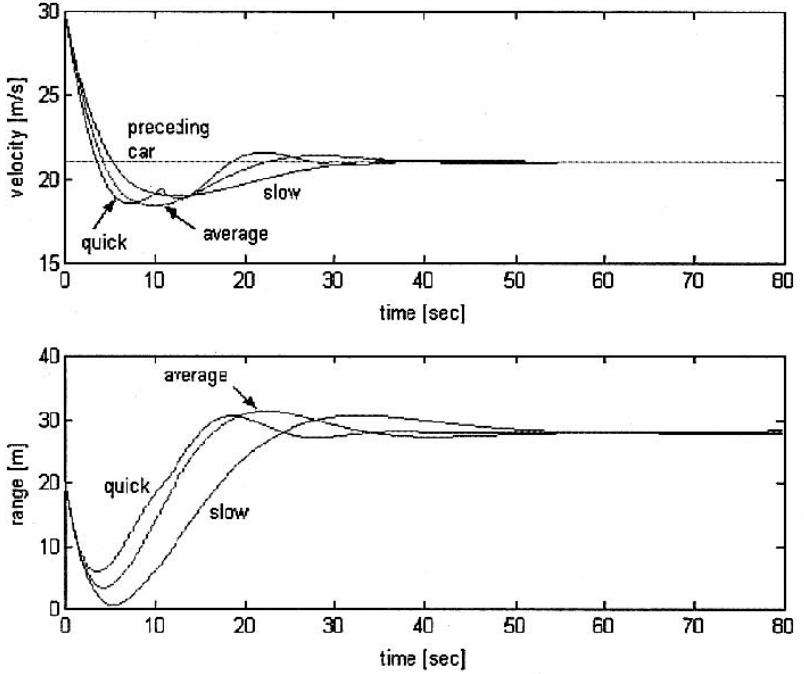

(b)
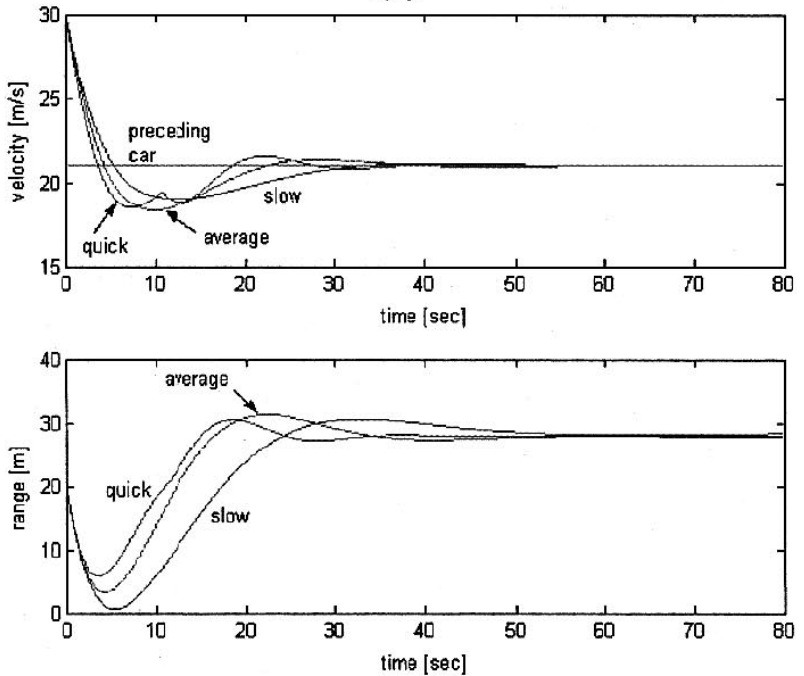

(d)

\section{TRAFFiC-Flow Simulation Methodology}

Preliminary analyzes of ACC string stability was done with Matlab/Simulink in the previous section, but to have more information on the effect of ACC systems on Highway traffic, a much more complicated simulation tool is needed.

In recent years, many microscopic simulation tools have been developed, such as AIMSUN [5], SmartAHS [6], TRANSIMS [7], CORSIM [8], etc. Microscopic simulators have many benefits over macroscopic ones. They recognize each vehicle/driver's personality, keep track of the motion of individual vehicles, thus allowing statistical outputs (distribution of Time to Collision, Velocity, etc.), and they can accept lane-changing models.

A microscopic vehicle simulation developed by Liang and Peng, 2000 [9] has been used in developing computer tools for evaluating the influences of ACC-equipped vehicles on traffic flow. A major advantage of this simulator is that two components of the human driver (a longitudinal model and a lateral model) are both constructed based on statistical analysis 

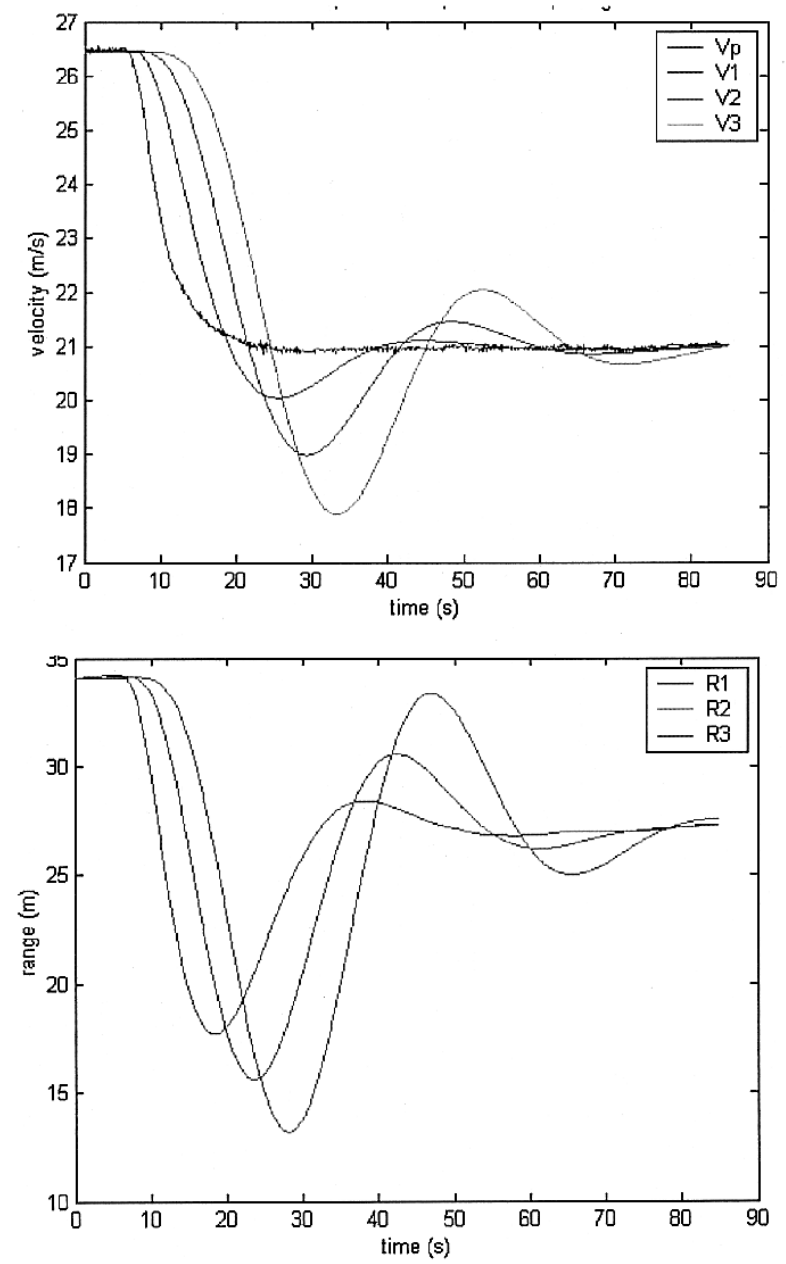

Fig. 8. Velocity and range clearance of a string of average ACC vehicles.

of human drivers' characteristics from field measurement work [1].

This simulation tool records the motions of several thousand vehicles on a two-lane circular track. The length of this track and the number of cars are limited only by simulation time constraints. In the simulation, vehicles change lanes when their drivers see opportunities to travel faster. This introduces perturbations into the traffic stream, thereby allowing string performance issues to occur.

The simulated cars can be a mixture of human-driven cars with and without ACC assistance. The influence of ACC vehicles on traffic flow can be examined by simulating different levels of penetration of ACC (number of ACC-equipped cars/total number of cars) and different traffic densities. In the following sections, the details of the human driver models used in the simulation are described.

\section{A. Human Driver Model}

In the last fifty years, many longitudinal human driver models have been proposed: Pipes [10], Newell [11], Gazis [12], Tyler [13], Gipps [14], and recently Bando [15]. These models were tested and compared by Kangwon Lee and Huei Peng [16], using the ICC FOT database [1] and the SAVME database [17]. A modified version of the Gipps model was also tested, and it was judged to provide the best fit to data measured in natural driving. The mathematical form of the modified Gipps model is shown in (4) at the bottom of the page where:

$a_{n} \quad$ Driver's maximum allowable acceleration, vehicle n;

$b_{n} \quad$ Driver's maximum allowable deceleration, vehicle $\mathrm{n}$;

$\hat{b} \quad$ Estimated value for $b_{n-1}$;

$s_{n} \quad$ The effective size of vehicle n: Actual size plus a margin;

$V_{n o} \quad$ Driver's desired speed, vehicle n;

$x_{n}(t)$ The location of the front of vehicle $\mathrm{n}$ at time $\mathrm{t}$;

$V_{n}(t)$ The speed of vehicle $\mathrm{n}$ at time $t$;

$\tau \quad$ The apparent reaction time.

The Gipps model satisfies two goals that are respectively represented by the two halves of the equation. The first half tends to accelerate to the desired free flow speed. The second half tends to keep a safe distance from the preceding car. The velocity at time $t+\tau$ is chosen to satisfy one of these two goals, whichever is more conservative.

Using the ICC-FOT database, Lee and Peng [16] found a statistical distribution of these parameters that was included in the simulation. To avoid collisions, an emergency braking logic was added to this model, applying $0.5 \mathrm{~g}$ deceleration when the range clearance is smaller than a certain minimum allowed range clearance.

\section{B. ACC Models}

The ACC models used in the UM-ACCSIM were according to the representative ACC systems described in Section V.A.

\section{Preliminary Traffic Flow Simulations}

Traffic simulations have been used to study different traffic densities and different ACC market-penetrations: $0 \%$ (i.e., only human drivers), $10 \%, 20 \%$, and 30\% [3], [16].

Detailed time histories of range clearance and velocity for the manually-driven vehicles reveal that the driver model produces accelerating and braking inputs that are more aggressive than what one would expect. This is perhaps due to the fact that these drivers are "full-time" drivers. That is, they pay attention to the driving task $100 \%$ of the time, and they have extraordinary performance characteristics.

These are preliminary results. They employ a human driver model that fits measured data on manual driving better than sev-

$$
V_{n}(t+\tau)=\min \left\{\begin{array}{l}
V_{n}(t)+2.5 \cdot a_{n} \cdot \tau \cdot\left(1-\frac{V_{n}(t)}{V_{n o}}\right) \sqrt{0.025+\frac{V_{n}(t)}{V_{n o}}} \\
b_{n} \cdot \tau+\sqrt{b_{n}^{2} \cdot \tau^{2}-b_{n} \cdot\left[2 \cdot\left(x_{n-1}(t)-s_{n-1}-x_{n}(t)-V_{n}(t) \cdot \tau\right)-\frac{V_{n-1}(t)^{2}}{\hat{b}}\right]}
\end{array}\right.
$$


eral other models. However, a very careful validation of transient response has not been conducted. Hence, the main conclusion derived from these preliminary simulation results, is a need to develop a better model of human-driving behavior. Although ACC performance can be characterized well, a simulation evaluation involving large percentages of manually-driven vehicles depends primarily upon the model employed to represent manual driving behavior.

Characterization of the microscopic qualities of manual driving is the subject of on-going research.

\section{CONCLUDING STATEMENTS}

Significant results were reported in this paper concerning the measurement and analysis of ACC system performance, with emphasis on their effect on traffic flow. As described in this paper, a retro-fit package suitable for the measurement of ACC vehicle performance has been used to assess the behavior of commercially-available ACC vehicles. Based on measured characteristics of ACC systems, simulation analyzes indicate that currently-available ACC-equipped vehicles will have string-performance qualities that are characterized by substantial overshoots in velocity and range clearance in response to changes in the velocity of the preceding vehicle. In terms of the state-space parameters identified for these vehicles, the linear transfer function for velocity from the $\mathrm{i}$-th vehicle to the $\mathrm{i}+1^{\text {st }}$ vehicle in a string is such that $\left|V_{i+1}(s) / V_{i}(s)\right|>1.0$ at frequencies typical of those encountered in highway driving.

These results are in agreement with earlier studies involving a prototypical ACC system [1]. The amplitude of perturbations in speed will be amplified from vehicle to vehicle in the stream. However, driver-controlled vehicles perform in a similar manner, and it is not clear whether ACC performance will interact with manual performance in a manner that will degrade traffic flow. A favorable ultimate outcome of this type of research may be the development of ACC systems that tend to improve traffic flow when traffic density is high.

The effect of these ACC vehicles on traffic flow is not completely known. Nevertheless, early results indicate that the characteristics of ACC vehicles will have a significant impact on traffic flow. Further development of the human driver model into one that is accurate enough for high-fidelity traffic simulation appears to be the most important next step for this research.

\section{REFERENCES}

[1] P. Fancher et al., "Intelligent Cruise Control (ICC) Field Operational Test," Final Report," Univ. Michigan Transportation Research Institute, Washington, DC, UMTRI-98-17, 1998.

[2] — "Research on Desirable Adaptive Cruise Control Behavior in Traffic Streams," First Phase Final Report," The Univ. of Michigan Transportation Research Institute (UMTRI), With Participation by Nissan Motor Company, Bayerische Motoren Werke AG (BMW), and Daimler/Chrysler AG, UMTRI-2002-16, 2002.

[3] H. Peng, "Evaluation of driver assistance systems - A human-centered approach," presented at the Keynote Paper, Proc. AVEC'02, 6th Int. Symp. Advance Vehicle Control, Hiroshima, Japan, Sept. 9-13, 2002.

[4] P. Fancher et al., "Evaluating the influences of adaptive cruise control systems on the longitudinal dynamics of strings of highway vehicles," presented at the Proc. 17th IAVSD Conf., Copenhagen, Denmark, 2001.
[5] J. Barceló, J. Casas, J. L. Ferrer, and D. García, "Modeling advanced transport telematic applications with microscopic simulators: The case of AIMSUN," in Proc. 10th Eur. Simulation Symp., 1998, pp. 362-367.

[6] M. Antoniotti and A. Göllü, "SHIFT and smartAHS: A language for hybrid systems engineering, modeling, and simulation," presented at the USENIX Conf. Domain Specific Languages, Santa Barbara, CA, Oct. 1997.

[7] L. L. Smith, "Status report on TRANSIMS development," presented at the Transportation Research Board 79th Ann. Meet., Washington, DC, Jan. 12, 2000.

[8] CORSIM Users Guide, vol. 3, TSIS 5.0 documentation, FHWA-TSIS Company, 2000.

[9] C. Liang and H. Peng, "String stability analysis of adaptive cruise controlled vehicles," JSME Int. J., ser. C, vol. 43, no. 3, pp. 671-677, Sept. 2000.

[10] L. A. Pipes, "An operational analysis of traffic dynamics," J. Appl. Phys., vol. 24, pp. 271-281, 1953.

[11] G. F. Newell, "Nonlinear effects in the dynamics of car following," Ops. Res., vol. 9, pp. 209-229, 1961.

[12] D. C. Gazis, R. Herman, and R. W. Rothery, "Nonlinear follow-theleader models of traffic flow," Ops. Res., vol. 9, pp. 545-566, 1961.

[13] J. S. Tyler, "The characteristics of model following systems as synthesized by optimal control," IEEE Trans. Automat. Control, vol. AC-9, pp. 485-498, 1964.

[14] P. G. Gipps, "A behavioral car-following model for computer simulation," Transport. Res. Board, vol. 15B, pp. 105-111, 1981.

[15] M. Bando et al., "Phenomenological study of dynamical model of traffic flow," J. Phys. I France, vol. 5, pp. 1389-1399, 1995.

[16] K. Lee and H. Peng, Identification and Verification of a Human Driving Model for Collision Warning and Control Systems. Ann Arbor, MI: Univ. Michigan, Mechanical Eng. Dept., to be published.

[17] R. Ervin et al., "System for Assessment of the Vehicle Motion Environment (SAVME)," Univ. Michigan Transportation Research Institute, UMTRI-2000-21-1, 2000

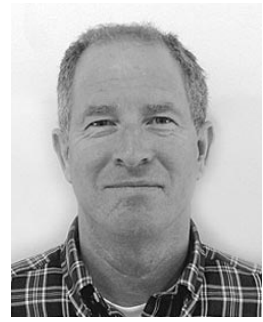

Zevi Bareket joined University of Michigan Transportation Research Institute (UMTRI), Ann Arbor, in 1989. Since then he has been involved in a variety of research projects focused about the dynamic performance characteristics of motor vehicles, as well as characterizing the way drivers operate these vehicles. In recent years, his research activities and interests were focused primarily on investigating ITS technologies aimed at enhancing highway safety and operations, as well as the development of driver models that represent the human operator. He has been involved with studies that addressed ITS approaches for collision avoidance and rollover prevention of heavy trucks. He studied the longitudinal an lateral performance of trucks, and the application of ITS technologies to traffic operations. Currently, he is primarily involved in ITS-related projects whose goal is to evaluate safety and performance of ITS technologies, and to expand the understanding of the driving process.

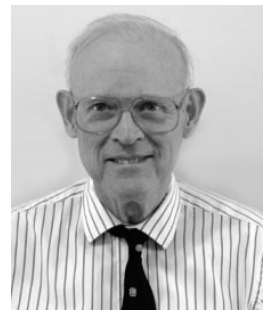

Paul S. Fancher received B.S.E., M.S.E., and Professional degrees from the University of Michigan, Ann Arbor.

He began his research career in the Analog Computer Laboratory at the University of Michigan in 1957. In 1967, he began working on highway safety and the control and stability of motor vehicles. This led to his research work at the University of Michigan Transportation Research Institute, Ann Arbor, on vehicle dynamics and the performance of the driver-vehicle-highway system. The underlying theme of his research is a performance prediction approach involving the application of simulation models, parameter measurement techniques, and vehicle testing and measurement procedures to the evaluation of existing and proposed vehicles and to the creation of new vehicle systems using advanced technology and control concepts. Currently, he is a Senior Research Scientist Emeritus at the University of Michigan. 


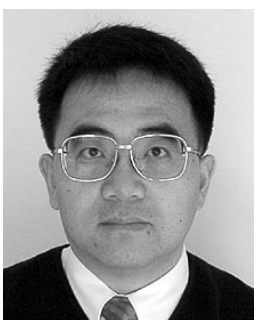

Huei Peng received the Ph.D. degree in mechanical engineering from the University of California, Berkeley, in 1992.

He is currently an Associate Professor in the Department of Mechanical Engineering, University of Michigan, Ann Arbor. His research interests include adaptive control and optimal control, with emphasis on their applications to vehicular and transportation systems.

Dr. Peng has been an active Member of the Society of Automotive Engineers (SAE) and the Dynamic System and Control Division, American Society of Mechanical Engineers (ASME), New York. He received the National Science Foundation (NSF) Career award in 1998. He has served as the chair of the ASME DSCD Transportation Panel from 1995 to 1997 . He is currently an Associate Editor for the IEEE/ASME TRANSACTIONS ON MECHATRONICS.

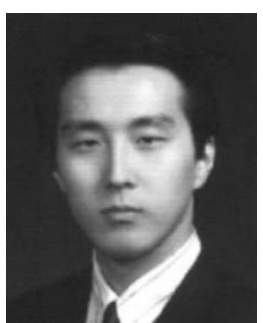

Kangwon Lee received the B.S. and M.S. degrees in mechanical engineering from Yonsei University, Seoul, South Korea, in 1993 and 1995, respectively, and the M.S. degree in electrical engineering/systems from the University of Michigan, Ann Arbor, in 2002, where he is currently working toward the Ph.D. degree in mechanical engineering.

He worked for the Hyundai Motor Company from 1995 to 1998 and is on academic leave until 2003. His research interests are intelligent transportation systems with emphases in adaptive cruise control systems and forward collision avoidance systems.

Charbel A. Assaf was born in Lebanon in 1979. He completed engineering studies at the Ecole Centrale Paris, France, in 2000 and received the M.S.E. degree in mechanical engineering with concentration on dynamics and control from the University of Michigan, Ann Arbor, in 2001.

Previously, he was a Research Assistant at the University of Michigan Transportation Research Institute, received training at BMW (Bayerische Motoren Werke), and currently works at Robert Bosch Corporation, Farmington Hills, MI 